\title{
Clinical Significance of 5-Fluorouracil Chemosensitivity Testing in Patients with Colorectal Cancer
}

\author{
WOONG BAE JI ${ }^{1}$, JUN WON UM ${ }^{1}$, JE SEOCK RYU ${ }^{1}$, KWANG DAE HONG ${ }^{1}$, JUNG SIK KIM ${ }^{1}$, \\ BYUNG WOOK MIN ${ }^{2}$, SUNG YEOP JOUNG ${ }^{2}$, JU HAN LEE ${ }^{3}$ and YOUNG SIK KIM ${ }^{3}$ \\ Departments of ${ }^{1}$ Surgery and ${ }^{3}$ Pathology, Korea University Ansan Hospital, Gyeonggi-do, Republic of Korea; \\ ${ }^{2}$ Department of Surgery, Korea University Guro Hospital, Seoul, Republic of Korea
}

\begin{abstract}
Aim: To evaluate whether the results of chemosensitivity testing were associated with prognosis of colorectal cancer patients after adjuvant 5-fluorouracil $(F U) /$ leucovorin chemotherapy. Patients and Methods: Eighty-nine patients who received 5-FU/leucovorin adjuvant chemotherapy for colorectal cancer were enrolled. Chemosensitivity tests were performed and tumor growth inhibition rate was calculated using the MTT (3-(4,5dimethylthiazol-2-yl)02,5-diphenyl-2H tetrazolium bromide) assay. Results: Fifty-one patients (57.3\%) were sensitive to 5-FU according to the chemosensitivity test. After a median follow-up of 64 months, there was a significant difference between the 5-year disease-free survival rates of the chemosensitive and chemo-resistant groups. However, there was no significant difference in the overall 5-year survival between the chemo-sensitive and chemo-resistant groups. Conclusion: A positive 5-FU sensitivity test with in vitro histoculture drug response assay (HDRA) was associated with better diseasefree survival. Chemosensitivity may be a prognostic factor for colorectal cancer patients undergoing adjuvant 5-FU/ leucovorin chemotherapy.
\end{abstract}

Although the survival rate of patients with colorectal cancer is increasing, this type of cancer remains one of the leading causes of cancer-related death (1). Surgery is the primary modality used to treat colorectal cancer and adjuvant therapy provides an additional survival benefit (2). Chemotherapy after curative resection of cancer is associated with a wide variety of outcomes. The ability to predict the response to

Correspondence to: Jun Won Um, MD, Ph.D., Division of Colon and Rectal Surgery, Department of Surgery, Korea University Ansan Hospital, 123 Jeokgeum-ro, Danwon-gu, Ansan, Gyeonggi province, 15355 Republic of Korea. Tel: +82 314124830, Fax: +82 314134829, e-mail: junwonum@korea.ac.kr

Key Words: Colorectal cancer, chemotherapy, chemosensitivity, 5fluorouracil, survival. certain chemotherapeutic agents would be helpful in a number of ways. Specifically, such predictive powers would provide valuable information for favorable outcomes and reduce complications caused by the chemotherapeutic agents. There have been several studies on various cancers examining the relationship between postoperative prognosis and chemosensitivity (3), as well as the usefulness and applicability of chemosensitivity testing in vitro. Furthermore, a number of studies have correlated clinical response with the results of chemosensitivity testing of malignancies; however, none of these have focused on colorectal cancer. Thus, the purpose of this study was to evaluate whether the results of chemosensitivity testing using the histoculture drug response assay (HDRA) were associated with the prognosis of colorectal cancer patients.

\section{Patients and Methods}

Patients and data. Prospectively collected data were analyzed retrospectively for patients with $\mathrm{T} 3$ or $\mathrm{T} 4$ colorectal cancer without nodal involvement and node-positive rectal cancer without distant metastasis, who had undergone radical resection and treatment with 5-fluorouracil (5-FU)/leucovorin adjuvant chemotherapy at the Korea University Ansan Hospital between December 2005 and December 2012. D2 or D3 lymphadenectomies were routinely performed for all patients. Pathologic examinations were conducted by experienced pathologists and the pathologic stage was determined according to the 7th Edition of the American Joint Committee on Cancer guidelines. Patients who had familial colon cancer, a history of other malignancies, as well as patients who underwent surgical resection other than R0 resection, were excluded from this study. Patients who previously had colorectal cancer and those who had received prior chemotherapy, immunotherapy or radiotherapy were also excluded. All patients included in the study received chemotherapy within 6 weeks of surgery. The Institutional Review Board of Korea University Ansan Hospital approved this study (AS16142-002).

Chemotherapy. All patients received 5-FU/leucovorin chemotherapy as an adjuvant treatment. The regimen used in this study consisted of an intravenous continuous infusion of $5-\mathrm{FU}\left(425 \mathrm{mg} / \mathrm{m}^{2}\right)$ plus leucovorin $\left(20 \mathrm{mg} / \mathrm{m}^{2}\right)$, daily for 5 days, every 4 weeks, over 6 cycles, after surgical resection. 
Follow-up. During chemotherapy, patients underwent routine follow-up examinations, including abdomen and pelvis computed tomography (CT), chest CT, carcinoembryonic antigen (CEA) measurement, routine blood analysis every 3 cycles and total colonoscopy after completion of chemotherapy. For patients who discontinued chemotherapy, routine follow-up examinations were performed after the last chemotherapy treatment and every 6 months after the operation for a total of a 2-year postoperative period, with the exception of the CEA measurement that was performed every 3 months at least for a postoperative period of 5 years.

HDRA with an MTT end-point. HDRA was performed according to the modified protocol of Hoffman et al. (4). Collagen gel sponges were cut into $10-\mathrm{mm}$ cubes and placed in the wells of a 24 -well plate. The sponges were then soaked with RPMI-1640 culture medium containing 20\% fetal bovine serum and 25x Fungizone (Gibco, Grand Island, NY, USA). The fresh surgical specimens were washed 3 times with RPMI-1640 medium and then cut into 24, 1-mm cubes. Next, a tissue cube was placed on each of the gel sponges in each well. After 24-hour incubation, 5-FU was added to the plate and tested in the culture medium at final concentrations of $3,12,6.25,12.5,25,50$ and $100 \mu \mathrm{g} / \mathrm{ml}$. Wells that did not contain the chemotherapeutic agent were used as a control. After culturing for another 6 days at $37^{\circ} \mathrm{C}$ in an atmosphere of $5 \% \mathrm{CO}_{2}, 5 \mathrm{mg}$ of 3-(4,5-dimethylthiazol-2-yl)02,5diphenyl-2H tetrazolium bromide (MTT; Sigma-Aldrich, St. Louis, MO, USA) was dissolved in $1 \mathrm{ml}$ of phosphate-buffered saline containing $100 \mathrm{nM}$ sodium succinate and $100 \mu \mathrm{l}$ of the resulting solution was added to each well. After incubation for 3 hours at $37^{\circ} \mathrm{C}$, the tissue cubes were transferred to new wells containing $1 \mathrm{ml}$ of dimethyl sulfoxide (DMSO) for extraction of the MTT-formazan product. Then, $100 \mu \mathrm{l}$ of the extract was transferred to each well of a 96-well plate and the absorbance of the wells was measured at 540 $\mathrm{nm}$ using a microplate reader. The wet weight of the tumor tissues was measured after extraction with DMSO. The tumor growth inhibition rate (IR) was calculated by the following equation: IR $(\%)=(1-$ mean absorbance $/ g$ of the drug-treated wells/mean absorbance/g of the control wells) $\times 100$. In this study, the tumor was regarded as sensitive to the chemotherapeutic agent when the IR was $\geq 30 \%$ at 5 -FU concentrations $>50 \mu \mathrm{g} / \mathrm{ml}(5,6)$.

Statistical analysis. Clinicopathological analysis and comparison of toxicities were performed using the Student's $t$-test and chi-square test. Survival was analyzed using the Kaplan-Meier method with the log-rank test. The Cox proportional hazards model was applied to identify recurrence risk factors. All statistical analyses were performed using SPSS, version 20 (IBM Corp., Armonk, NY, USA).

\section{Results}

Of the 89 patients enrolled in this study, 51 (57.3\%) were sensitive to 5-FU according to the chemosensitivity test results. No significant differences between the 5-FU chemosensitive and 5-FU chemo-resistant groups were observed with respect to sex, body mass index (BMI), tumor size, tumor location, preoperative CEA levels, T-stage, $\mathrm{N}$-stage, tumor emboli and number of retrieved lymph nodes (Table I).

After a median follow-up of 64 months, there was a statistically significant difference between the 5-year disease-free survival rates of the chemo-sensitive and
Table I. Clinicopathological characteristics of the 5-fluorouracil (5-FU) chemo-sensitive and chemo-resistant groups.

\begin{tabular}{|c|c|c|c|}
\hline & $\begin{array}{l}\text { 5-FU chemo- } \\
\text { sensitive } \\
(n=51)\end{array}$ & $\begin{array}{l}\text { 5-FU chemo- } \\
\text { resistant } \\
(\mathrm{n}=38)\end{array}$ & $p$-Value \\
\hline Age $($ median \pm SD) & $63.0 \pm 9.6$ & $69.0 \pm 11.0$ & 0.125 \\
\hline Gender, n (\%) & & & 0.476 \\
\hline Male & $27(52.9)$ & $23(60.5)$ & \\
\hline Female & $24(47.1)$ & $15(39.5)$ & \\
\hline BMI $(>25)$ & $16(31.4)$ & $14(36.8)$ & 0.589 \\
\hline ASA, n (\%) & & & 0.686 \\
\hline 1 & $20(39.2)$ & $15(39.5)$ & \\
\hline 2 & $30(58.8)$ & $21(55.3)$ & \\
\hline 3 & $1(2.0)$ & $2(5.3)$ & \\
\hline Tumor size $($ median \pm SD $)$ & $5.0 \pm 1.6$ & $5.0 \pm 1.8$ & 0.147 \\
\hline Location, n (\%) & & & 0.922 \\
\hline Colon & $22(43.1)$ & $16(42.1)$ & \\
\hline Rectum & $29(56.9)$ & $22(57.9)$ & \\
\hline Operation method, n (\%) & & & 0.072 \\
\hline Open & $38(74.5)$ & $21(55.3)$ & \\
\hline Laparoscopy & $13(25.5)$ & $17(44.7)$ & \\
\hline Preoperative CEA, n (\%) & & & 0.761 \\
\hline$>5$ & $11(21.6)$ & $7(18.9)$ & \\
\hline$<5$ & $40(78.4)$ & $30(81.1)$ & \\
\hline $\mathrm{T}$ stage, $\mathrm{n}(\%)$ & & & 0.305 \\
\hline 1 & 0 & $1(2.6)$ & \\
\hline 2 & $2(3.9)$ & $4(10.5)$ & \\
\hline 3 & $48(94.1)$ & $33(86.8)$ & \\
\hline 4 & $1(2.0)$ & 0 & \\
\hline $\mathrm{N}$ stage, $\mathrm{n}(\%)$ & & & 0.062 \\
\hline 0 & $39(76.5)$ & $19(50.0)$ & \\
\hline 1 & $8(15.7)$ & $14(36.8)$ & \\
\hline 2 & $4(7.8)$ & $5(13.2)$ & \\
\hline Tumor emboli, n (\%) & $12(23.5)$ & $12(31.6)$ & 0.397 \\
\hline $\begin{array}{l}\text { Retrieved lymph nodes } \\
(\text { median } \pm \text { SD) }\end{array}$ & $29.0 \pm 12.9$ & $25.0 \pm 16.3$ & 0.543 \\
\hline
\end{tabular}

SD, Standard deviation; BMI, body mass index; ASA, American Society of Anesthesiologists; CEA, carcinoembryonic antigen.

chemo-resistant groups; $88.7 \%$ and $68.9 \%$, respectively $(p=0.025)$. However, there was no significant difference in the overall 5-year survival between the chemo-sensitive and chemo-resistant groups $(95.9 \%$ vs. $85.1 \% ; p=0.233$; Figure 1).

A Cox proportional hazards model was applied to identify risk factors for disease-free survival in the two groups. Several factors were included in this model, including node metastasis, tumor emboli, age, preoperative CEA levels, BMI and 5-FU chemosensitivity. Significant risk factors were 5-FU chemosensitivity and preoperative CEA levels. After adjusting for these risk factors, only 5FU chemosensitivity remained significant in the hazards model. The adjusted hazard ratio was 0.274 for $5-\mathrm{FU}$ chemosensitivity $(95 \%$ confidence interval $(\mathrm{CI})=0.095$ 0.793; Table II). 


\section{5-year Overall Survival}

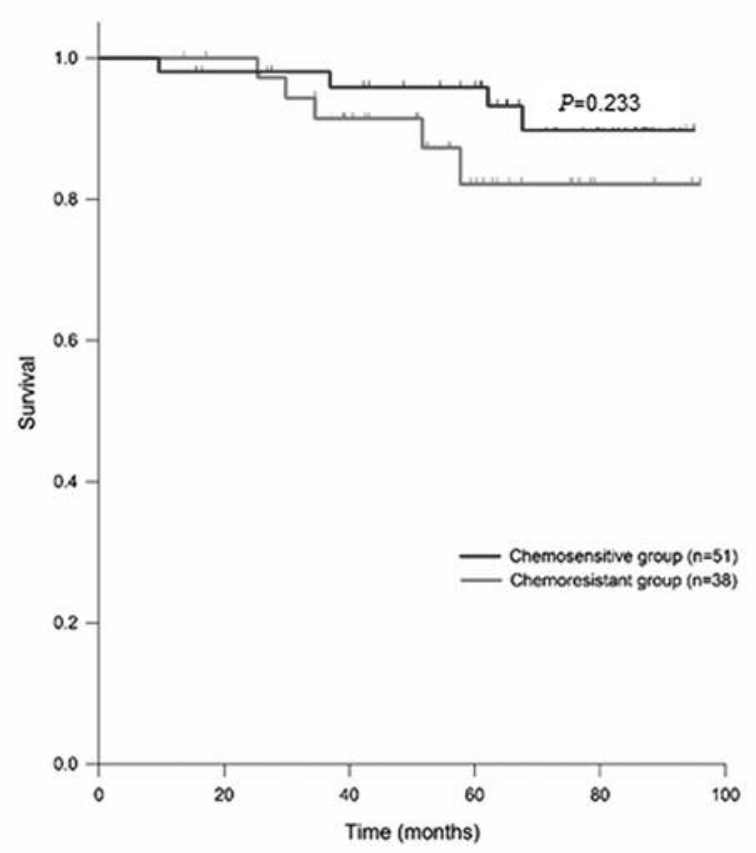

5-year Disease-free Survival

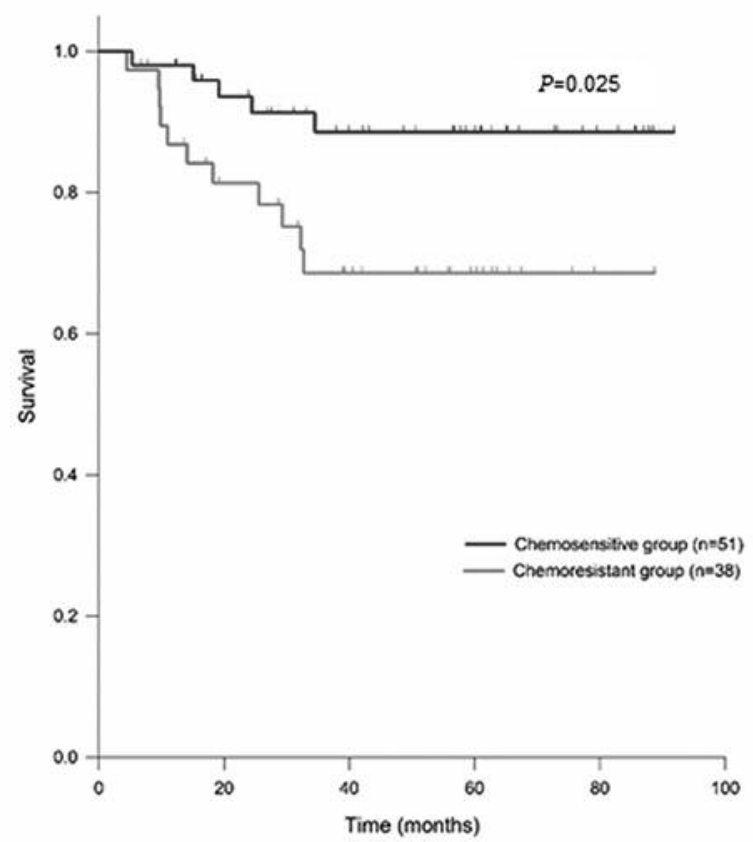

Figure 1. Kaplan-Meier survival analysis of the chemo-sensitive and chemo-resistant groups; there was a statistically significant difference for disease-free survival, but not for overall survival.

\section{Discussion}

In this study, we found that patients in the chemo-resistant group had a poorer disease-free survival rate than those in the chemo-sensitive group. To the best of our knowledge, this is the first study to compare survival differences in colorectal cancer patients on the basis of chemosensitivity status. Although Yoon et al. reported that clinical response correlates with HDRA chemosensitivity results (5); measurement of the clinical response in their report was limited to patients with a recurrent cancer lesion or metastatic lesion from radiologic studies, rather than survival data. Nevertheless, they concluded that the chemosensitivity test results were closely correlated with clinical response, with correlation rates of $66.3 \%$ for the FOLFOX regimen and $72.7 \%$ for the FOLFIRI regimen (5).

Several studies have identified a relationship between chemosensitivity and disease prognosis in some malignancies other than colorectal cancer. Jung et al. reported in their study of epithelial ovarian cancer that patients with lesions sensitive to taxane and carboplatin have a better progression-free survival (7). Similarly, Fujita et al. reported that for esophageal cancer patients who had preoperative concurrent chemoradiotherapy or surgery, chemo-sensitive patients had a better overall survival rate
Table II. Cox proportional hazards model for risk factors of recurrence.

\begin{tabular}{lcccc}
\hline & $\begin{array}{c}\text { Hazard } \\
\text { ratio }\end{array}$ & \multicolumn{2}{c}{$\begin{array}{c}\text { 95\% Confidence } \\
\text { interval }\end{array}$} & \\
\cline { 3 - 4 } & & Lower & Upper & \\
\cline { 3 - 4 } & & Lalue & \\
\hline 5-Fluorouracil chemosensitivity & 0.274 & 0.095 & 0.793 & 0.017 \\
Preoperative CEA & 2.668 & 0.965 & 7.371 & 0.058 \\
\hline
\end{tabular}

CEA, Carcinoembryonic antigen.

than those who were not chemo-sensitive, although this result was not statistically significant because of the small numbers involved (3). However, the American Society of Clinical Oncology (ASCO) Technology Assessment: Chemotherapy Sensitivity and Resistance Assays does not recommend routine application of chemosensitivity test to determine the chemotherapeutic agents for the patients with any kind of malignancies except clinical trial settings (8). Nevertheless, recently, two clinical trials for gastric cancer patients showed that the chemosensitivity test could predict the outcome of patients undergoing chemotherapy for advanced gastric cancer and it deserved further evaluation in the setting of prospective randomized trial $(9,10)$. 
Chemosensitivity to certain chemotherapeutic agents can be evaluated by using tests, such as the MTT assay, HDRA and collagen gel droplet-embedded culture drug-sensitivity test (11-13). HDRA was first reported by Hoffman et al. and is a three-dimensional culture system in which cell-to-cell contact is maintained so that long-term culture is possible under conditions resembling those in vivo (14). Importantly, this culture method makes it possible to evaluate the chemosensitivity of chemotherapeutic agents that are timedependent, such as 5-FU. In addition, modification of HDRA can be performed to identify cell viability using the MTT assay instead of autoradiography (4).

In our study, the overall survival of the chemo-sensitive group was better than that of the chemo-resistant group, although this did not reach statistical significance $(p=0.233)$. However, the number of patients included in this study was small and consisted primarily of stage II colon cancer; thus, the survival rate was already quite high $(95.9 \%$ vs. $85.1 \%)$, which may explain why there was no difference in the overall survival rate. The standard adjuvant chemotherapeutic agents for non-metastatic colorectal cancer with lymph node involvement are oxaliplatin-based. Thus, we are currently collecting survival data for chemosensitivity to oxaliplatin.

In conclusion, chemosensitivity testing using HDRA with an MTT end-point can be used to predict prognosis in patients with colorectal cancer treated with adjuvant 5-FU chemotherapy. Prospective studies are needed to determine if HDRA results are a useful prognostic factor.

\section{Conflicts of Interest}

We have no potential or actual personal, political or financial conflicts of interest to declare.

\section{References}

1 Siegel R, Naishadham D and Jemal A: Cancer statistics, 2013. CA Cancer J Clin 63: 11-30, 2013.

2 Chau I and Cunningham D: Adjuvant therapy in colon cancerwhat, when and how? Ann Oncol 17: 1347-1359, 2006.

3 Fujita Y, Hiramatsu M, Kawai M, Nishimura H, Miyamoto A and Tanigawa N: Histoculture drug response assay predicts the postoperative prognosis of patients with esophageal cancer. Oncol Rep 21: 499-505, 2009.

4 Furukawa T, Kubota T and Hoffman RM: Clinical applications of the histoculture drug response assay. Clin Cancer Res 1: 305311, 1995.

5 Yoon YS, Kim CW, Roh SA, Cho DH, Kim GP, Hong YS, Kim TW, Kim MB and Kim JC: Applicability of histoculture drug response assays in colorectal cancer chemotherapy. Anticancer Res 32: 3581-3586, 2012.
6 Kim JC, Shin ES, Kim CW, Roh SA, Cho DH, Na YS, Kim TW, Kim MB, Hyun YL, Ro S, Kim SY and Kim YS: In vitro evaluation of histone deacetylase inhibitors as combination agents for colorectal cancer. Anticancer Res 29: 3027-3034, 2009.

7 Jung PS, Kim DY, Kim MB, Lee SW, Kim JH, Kim YM, Kim YT, Hoffman RM and Nam JH: Progression-free survival is accurately predicted in patients treated with chemotherapy for epithelial ovarian cancer by the histoculture drug response assay in a prospective correlative clinical trial at a single institution. Anticancer Res 33: 1029-1034, 2013.

8 Burnstein HJ, Mangu PB, Seomerfield MR, Schrag D, Samson D, Holt L, Zelman D and Ajani JA: American Society of Clinical Oncology Clinical Practice Guideline update on the use of chemotherapy sensitivity and resistance assays. J Clin Oncol 29(24): 3328-3330, 2011.

9 Naitoh H, Yamamoto H, Murata S, Kobayashi H, Inoue K and Tani T: Stratified phase II trial to establish the usefulness of the collagen gel droplet embedded culture-drug sensitivity test (CDDST) for advanced gastric cancer. Gastric Cancer 17: 630-637, 2014.

10 Tanigawa N, Yamaue H, Ohyama S, Sakuramoto S, Inada T, Kodera Y, Kitagawa Y, Omura K, Terashima M, Sakata Y, Nashimoto A, Yamaguchi T, Chin K, Nomura E, Lee SW, Takeuchi M, Fujii M and Nakajima T: Exploratory phase II trial in a multicenter setting to evaluate the clinical value of a chemosensitivity test in patients with gastric cancer (JACCROGC 04, Kubota memorial trial). Gastric Cancer 19: 350-360, 2016.

11 Carmichael J, DeGraff WG, Gazdar AF, Minna JD and Mitchell JB: Evaluation of a tetrazolium-based semiautomated colorimetric assay: Assessment of chemosensitivity testing. Cancer Res 47: 936-942, 1987.

12 Inaba M, Tashiro T, Sato S, Ohnishi Y, Tanisaka K, Kobayashi $\mathrm{H}$ and Koezuka M: In vitro-in vivo correlation in anticancer drug sensitivity test using AUC-based concentrations and collagen gel droplet-embedded culture. Oncology 53: 250-257, 1996.

13 Hoffman RM: Three-dimensional histoculture: Origins and applications in cancer research. Cancer Cells 3: 86-92, 1991.

14 Kim R, Emi M, Tanabe K, Uchida Y and Toge T: Chemosensitivity testing for gastrointestinal cancer: Survival benefit potential and limitations. Anticancer Drugs 14: 715-723, 2003. 